\title{
Solar Energetic Particles measured by AMS-02
}

\author{
V. Bindi* \\ Physics and Astronomy Department, University of Hawaii at Manoa, 96822, HI, US \\ E-mail: bindi@hawaii.edu
}

\section{AMS-02 collaboration}

The Alpha Magnetic Spectrometer (AMS-02), with its acceptance of about $0.45 \mathrm{~m}^{2} \mathrm{sr}$, is the largest Solar Energetic Particle (SEP) detector in space. AMS-02 was installed on the International Space Station (ISS) on May 19, 2011, where it will take data for the duration of the station, currently extended till 2024. During the first three years of operation, AMS-02 measured increases in the proton flux near $1 \mathrm{GV}$ and above associated with twenty of the highest energy solar events, produced during $\mathrm{M}$ - and X-class flares and fast coronal mass ejections (CME) magnetically well-connected with the Earth. Thanks to its large acceptance and particle detection capabilities, AMS-02 is able to perform precise measurements in a short period of time which is typical of these transient phenomena and collect enough statistics to measure fine structures and time evolution of the proton spectrum. The events observed by AMS-02 since the beginning of its mission will be presented and some preliminary results will be shown. AMS-02 observations of protons and helium, with their unprecedented resolution and high statistics, will improve the understanding of SEP behavior at high energies and constrain models of SEP production.

The 34th International Cosmic Ray Conference,

30 July- 6 August, 2015

The Hague, The Netherlands

${ }^{*}$ Speaker. 


\section{The Need for Precise SEP Measurements above $\sim 500 \mathrm{MeV} /$ nucleon}

Solar Energetic Particles (SEPs) are a key target of Heliophysics research, not only as exemplars of particle acceleration and transport processes that are ubiquitous in astrophysical plasmas, but also as the most intense transient radiation hazard for human and robotic space-explorers. The energy range of SEPs extends over more than five orders of magnitude and SEP fluxes can vary by eight orders of magnitude, thus their energy spectrum has never been measured by a single instruments. SEPs are very well-observed by spacecraft covering particle energies below several hundred $\mathrm{MeV} /$ nucleon. Multiple missions, stretching back over decades, have yielded a fairly complete description of SEP intensity, energy spectra, and composition up to a few hundred MeV/nucleon. But SEP characteristics at even higher energies are, by comparison, only poorly understood due to the relative dearth of high-energy measurements. This situation is particularly unfortunate because these very high-energies are those at which the acceleration process begins to fail, as it becomes harder to confine the particles to the acceleration region. Seeing exactly how SEP spectra steepen at these energies - and how that steepening evolves during the event, how it differs among species, and how the steepening varies from one event to another - offers a largely unexploited and powerful opportunity to test and to develop our understanding of particle acceleration processes in the corona.

Because of limited satellite data, our information on SEPs above $\sim 500 \mathrm{MeV} /$ nucleon comes primarily from neutron-monitors (NMs) in Ground-Level Enhancements (GLEs). The weakness of such indirect measurements is that NMs can only detect secondary particles resulting from spallation events, making it impossible to identify the primary particles that initiated the air shower. It should also be noted that NMs provide no information on the simultaneous spectra of other species. Comparison of proton and helium spectra provide indeed additional insights into the acceleration processes and structure of the shock as pointed out by Lee (2005) [1].

As detailed below, the Alpha Magnetic Spectrometer (AMS-02) aboard the International Space Station (ISS) can provide measurements of SEP protons and helium at these very high energies.

\subsection{Excess in the Primary Proton Flux Observed by the AMS-02 experiment}

AMS-02 [2] is a state-of-the-art magnetic spectrometer that measures all species of particles in the energy range from hundreds of $\mathrm{MeV}$ to a few TeV. The AMS-02 experiment, in addition to studying phenomena such as dark matter and antimatter, measures cosmic rays continuously over the entire solar cycle. The AMS-02 experiment has the potential to make a unique contribution to the field of heliophysics by providing unprecedented measurements of solar protons and helium in the highest energy range of solar particles, generating spectra and their temporal evolution with superior resolution both in energy and time.

AMS-02 has a built-in redundancy across five different detectors, which measure the trajectory, charge, energy and velocity of electrons and nuclei up to Fe and above to very high accuracy. AMS02 is orbiting onboard the ISS between $\pm 52^{\circ}$ latitude, with particle rates that vary from $200 \mathrm{~Hz}$ close to the equator to $2000 \mathrm{~Hz}$ close to the geomagnetic poles or the South Atlantic Anomaly. Because of its large acceptance $\left(4500 \mathrm{~cm}^{2} \mathrm{sr}\right)$ and fast trigger rate, it is possible for AMS-02 to measure the variability of incoming particles on short timescales. 
Protons which cross through all of the AMS-02 sub-detectors, providing high quality measurements of charge, velocity, energy, and mass, and above the geomagnetic cutoff are selected [2]. The geomagnetic cutoff is calculated by backtracing particles from the top of AMS out to 50 Earth radii using the International Geomagnetic Reference Field (IGRF) [3] as described in [4]. At high geomagnetic latitudes, closer to the polar regions, the geomagnetic cutoff is very low, and most protons measured by AMS-02 are above it. At low geomagnetic latitudes, closer to the equatorial regions, the geomagnetic cutoff is higher and can reach up to about $20 \mathrm{GV}$. SEPs typically have rigidities below $2 \mathrm{GV}$ and thus can only be observed by AMS-02 near the poles when the geomagnetic cutoff is below this value, typically geomagnetic latitude $\left|\theta_{m}\right| \gtrsim 50^{\circ}$, corresponding to $\approx 20 \%$ to the total orbiting time. Maximum values of the IGRF cutoff calculated in the AMS-02 field of view as function of the latitude and longitude are shown in Fig. 1. The proton flux is then computed as described in [2].

Fig. 2 shows AMS-02 proton increase integrated over 24 hours measured during three very intense solar events in January 28th (blue crosses), March 7th (red rhombuses) and May 17th (green triangles), 2012 together with the GCR background flux (black dots) measured during a day of quiet sun. The excess of particles in AMS-02 fluxes is evident and well defined at rigidities below $2 \mathrm{GV}$ ( $\sim 1.2 \mathrm{GeV} /$ nuc). It is worth pointing out that all these three events in principle, could have been detected on ground if the sensitivity of NMs was higher, instead only one of those events was a GLE (May 17th, 2012) while the other two events did not provide a measurable excess in the counting rate of NMs.

\subsection{Observation of SEPs by AMS-02}

So far in Solar Cycle 24, only one official GLE was observed on May 17th, 2012 while another possible GLE on January 6th, 2014 was detected by South Pole neutron monitor and a few other

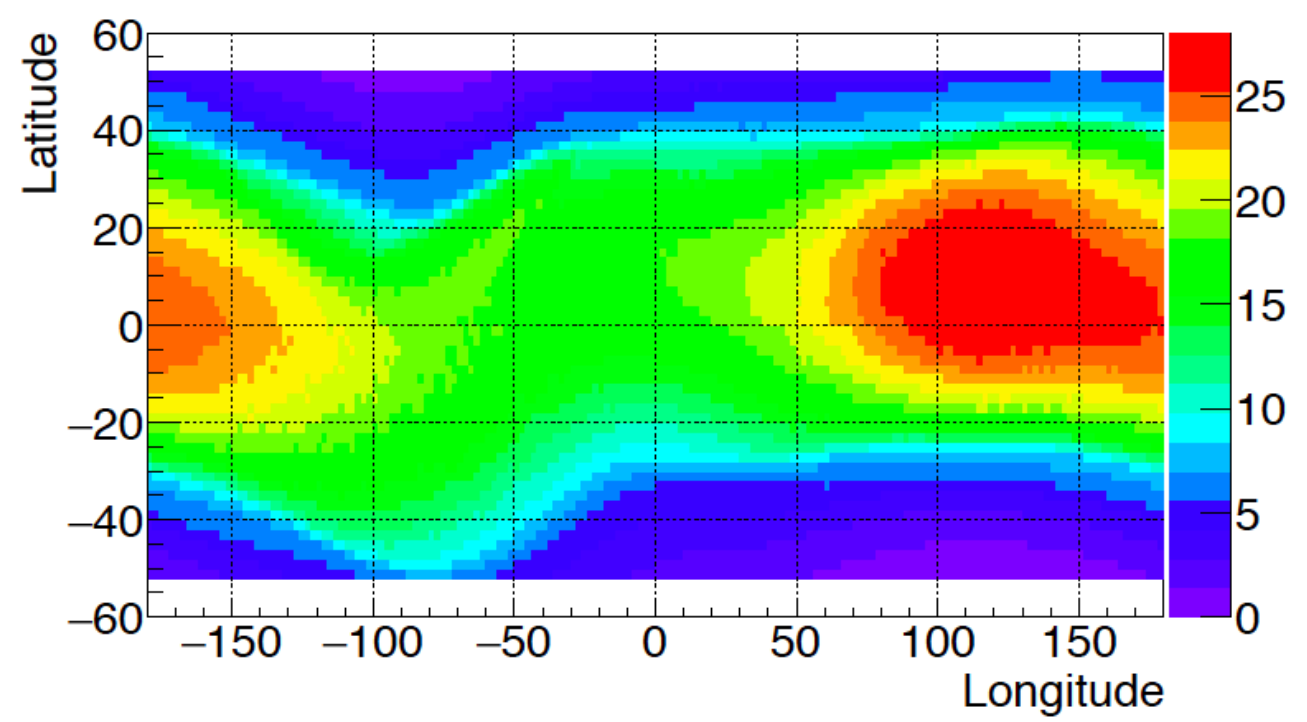

Figure 1: Values at different latitudes and longitudes of the maximum IGRF cutoff calculated in the AMS-02 field of view. 


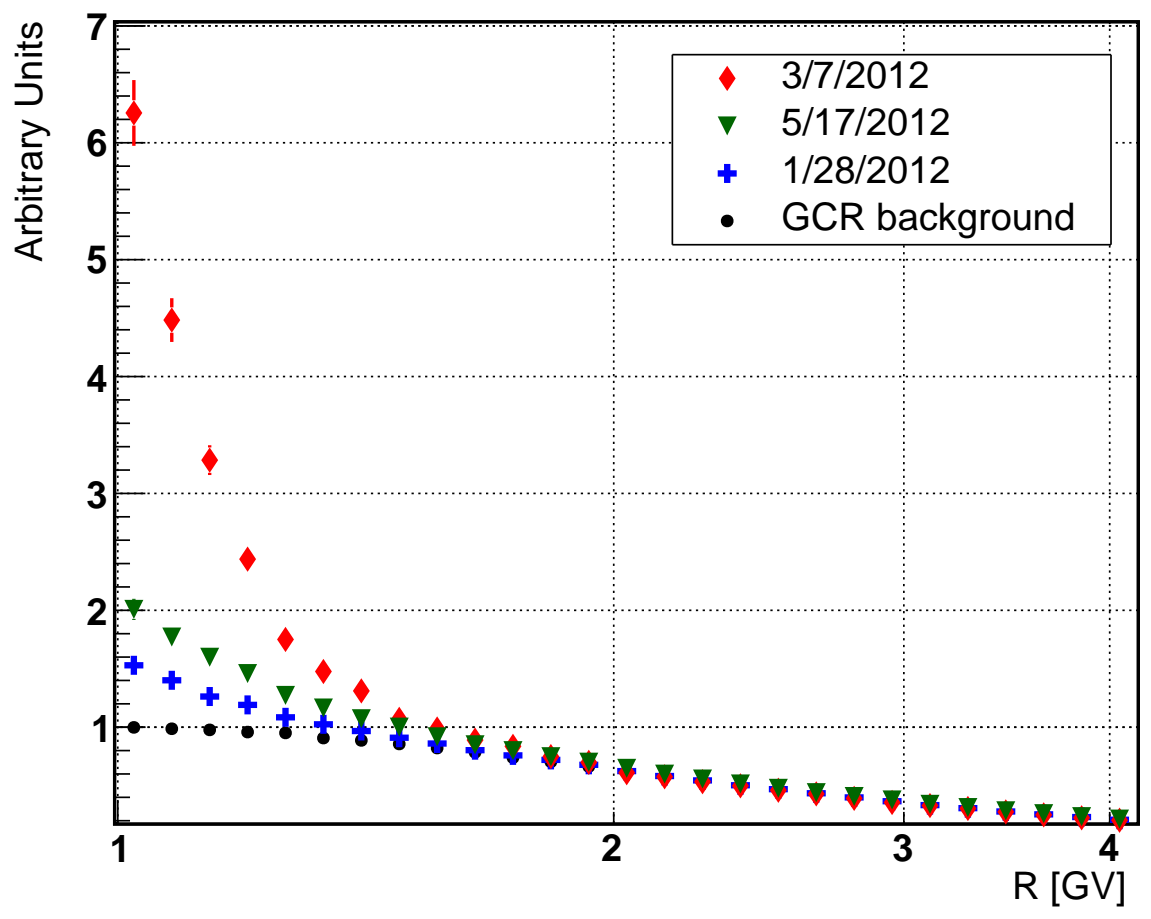

Figure 2: AMS-02 protons able the geomagnetic cutoff integrated over 24 hours measured during January 28th (blue crosses), March 7th (red rhombuses) and May 17th (green triangles), 2012 plotted with the GCR background (black dots). A rise related to the intense solar events is clearly visible at rigidities below 2 GV.

NMs in the southern hemisphere. In comparison, 7 GLEs were observed during Solar Cycle 23 by this point. It is common knowledge that the current solar cycle has been weaker with respect to the previous one. In [5], Gopalswamy et al. observe that the number of large SEP events in Cycle 24 is similar to that in Cycle 23, being 31 and 37 respectively. However, observations by GOES-13 in the high energy proton channels suggest that there were 12 SEP events with energies above 500 $\mathrm{MeV}$ in Cycle 23 compared to 5 in Cycle 24.

A list of events associated with a proton increase observed in AMS-02 at rigidities near $1 \mathrm{GV}$ $(\approx 430 \mathrm{MeV})$ and above caused by strong solar events occurred from June 2011 until December 2014 is presented in Table 1. All the increases in AMS-02 were associated with M- and X-class flares and fast CMEs. Depending on the events, the increases in AMS-02 lasted on the order of hours to days and reached different rigidities, typically below $2 \mathrm{GV}$. The most intense event of Cycle 24 so far began on March 7th, 2012 and was followed by two more major flares and high speed CMEs on March 9th and March 10th generating a continuous proton increase in AMS-02 for many days. The maximum energy for each event [5] measured by GOES is also indicated in the last column of the tabular. 


\begin{tabular}{|c|c|c|c|c|}
\hline $\begin{array}{c}\text { AMS-02 } \\
\text { Event }\end{array}$ & $\begin{array}{c}\text { Solar Event } \\
\text { Date }\end{array}$ & $\begin{array}{c}\text { Flare } \\
\text { Class }\end{array}$ & $\begin{array}{c}\text { CME } \\
\text { Vel. }(\mathbf{k m} / \mathbf{s})\end{array}$ & $\begin{array}{c}\text { GOES Max } \\
\text { E }(\mathbf{M e V})\end{array}$ \\
\hline 1 & $06 / 07 / 11$ & M2.5 & 1255 & $350-420$ \\
2 & $08 / 04 / 11$ & M9.3 & 1315 & $165-500$ \\
3 & $08 / 09 / 11$ & X6.9 & 1610 & $350-420$ \\
4 & $01 / 23 / 12$ & M8.7 & 2175 & $165-500$ \\
5 & $01 / 27 / 12$ & X1.7 & 2508 & $\mathbf{5 1 0}-\mathbf{7 0 0}$ \\
6 & $03 / 07 / 12$ & X5.4, X1.3 & 2684,1825 & $\mathbf{5 1 0}-\mathbf{7 0 0}$ \\
7 & $03 / 13 / 12$ & M-class & 1884 & $\mathbf{4 2 0}-\mathbf{5 1 0}$ \\
8 & $05 / 17 / 12$ & M5.1 & 1582 & $>\mathbf{7 0 0}$ \\
9 & $07 / 19 / 12$ & M7.7 & 1631 & $165-500$ \\
10 & $07 / 23 / 12$ & - & 2003 & $165-500$ \\
11 & $04 / 11 / 13$ & M6.5 & 861 & $165-500$ \\
12 & $05 / 22 / 13$ & M5.8 & 1466 & $350-420$ \\
13 & $10 / 28 / 13$ & M5.1, M2.8, M4.4 & $1201,1073,812$ & - \\
14 & $11 / 02 / 13$ & C8.2* & 828 & - \\
15 & $12 / 28 / 13$ & - & 1118 & $80-165$ \\
16 & $01 / 06 / 14$ & - & 1118 & $>\mathbf{7 0 0}$ \\
17 & $01 / 07 / 14$ & X1.2 & 1830 & $350-420$ \\
18 & $02 / 25 / 14$ & X4.9 & 2147 & - \\
19 & $09 / 01 / 14$ & - & 1404 & - \\
20 & $09 / 10 / 14$ & X1.6 & 1267 & - \\
\hline & & & & \\
\hline
\end{tabular}

Table 1: List of intense solar events between May 2011 and Feb 2014 associated with a proton increase observed by AMS-02 near $1 \mathrm{GV}$ and above. The columns represent: the number of event observed by AMS-02, the solar event date, the flare class, the CME velocity, the maximum energy detected by GOES-13 satellite [5] (events with energies above $500 \mathrm{MeV}$ are highlighted in bold). Information was not available for some flares. *The C-class flare is likely underestimated because it occurred at the sun's limb.

\subsection{Time evolution of SEPs}

Because AMS-02 has such a large acceptance $\left(4500 \mathrm{~cm}^{2}\right.$-sr), it is capable of achieving high statistics for short integration times, typically on the order of a few minutes, and study the time evolution of the increase. Fig. 3 shows the GCR background (black dots), calculated by integrating the flux of protons measured on the first quiet sun day prior to the solar event (May 16th, 2012), and the May 17th increase measured during short time intervals when AMS-02 was at high geomagnetic latitudes. Different colors represent different AMS-02 orbits. Fig. 3 clearly demonstrates that the high accuracy of the measurements over such a short period of time will allow detailed studies of the time evolution of the event.

\subsection{Multi-Spacecraft Observations to Build a Complete Picture of SEPs}

The energy range of SEPs extends over more than five orders of magnitude, thus their energy spectrum can only be measured by multiple instruments. The measurement of SEP spectral shapes at all energies allows for investigation of acceleration and transport processes pertinent to each energy range. Models that describe particle acceleration at the sun can be better defined by including precision high energy measurements from AMS-02, and thus result in more accurate un- 


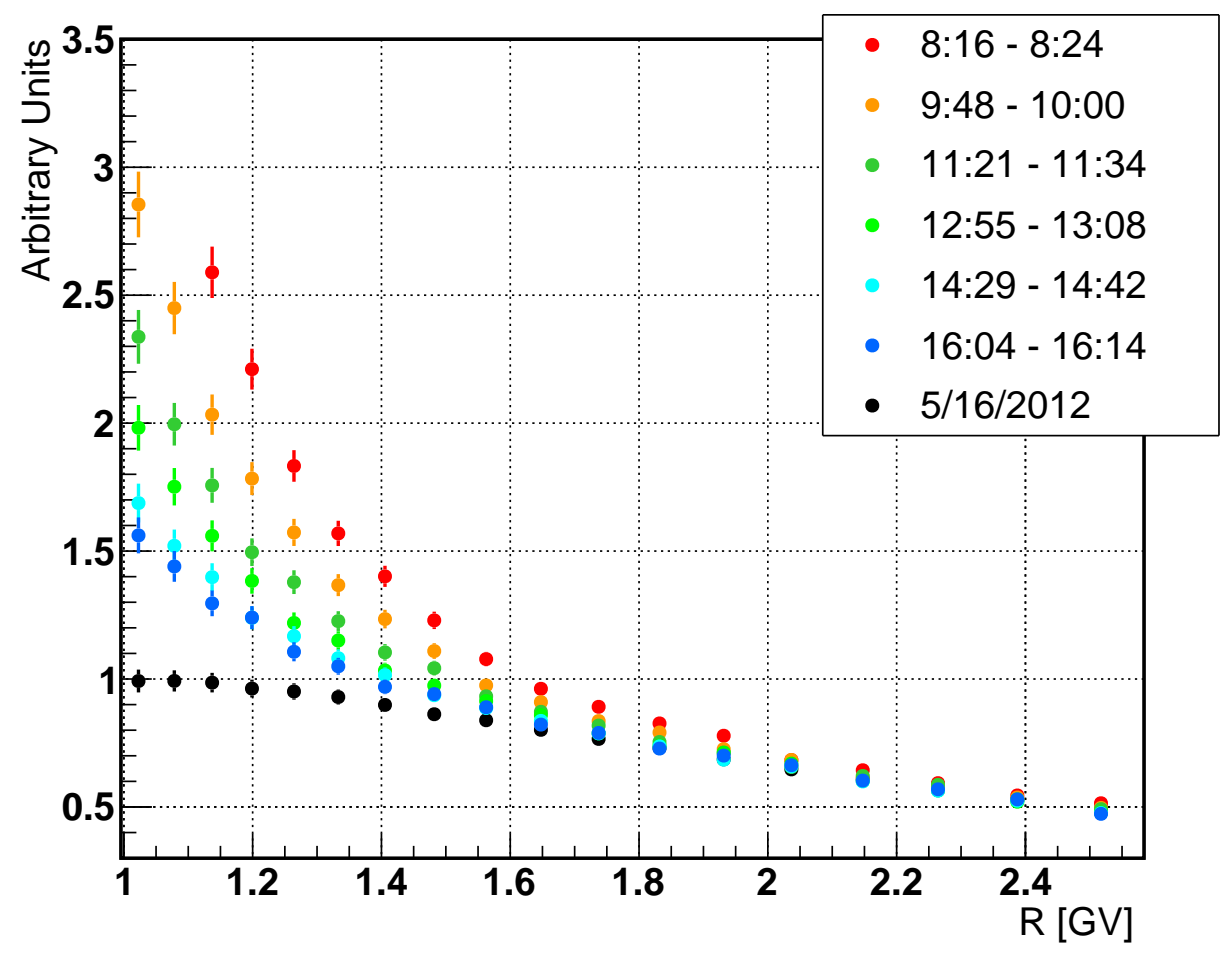

Figure 3: AMS-02 protons above cutoff integrated over 24 hours measured on May 16th, 2012 (GCR background, black) with spectra of May 17th, 2012 at different time intervals measured near the polar regions in different orbits (colors). Even in a few minutes integration time, the protons measured on May 17th show a clear increase at low rigidities with respect to the GCR background of a quiet sun day.

derstanding of the conditions inside of acceleration regions. Data from the GOES-13 satellite [6] are particularly suited to compare with AMS-02 because HEPAD (above $0.5 \mathrm{GV}$ ) has channels that overlap with AMS-02's lower rigidity range, while EPEAD (below $0.5 \mathrm{GV}$ ) extends to even lower rigidities that AMS-02 is not capable of measuring. Protons measured by GOES-13 HEPAD and EPEAD instruments are shown in Fig. 4. GOES-13 HEPAD and EPEAD proton fluxes measured in March 16 (empty dots) are plotted together with the fluxes integrated in the same period of time during May 17th event while AMS was orbiting near the North Geomagnetic Pole. The increases measured by GOES-13/EPEAD at rigidities below $0.5 \mathrm{GV}$ are particularly evident instead the value of the increase in the GOES-13/HEPAD channels is less obvious.

In Fig. 5, AMS-02 (left) and GOES-13/HEPAD (right) fluxes measured during the same periods of time in May 17th, 2012 and normalized to a day of quiet sun (May 16th) are shown. The increase is extremely clear in AMS-02 reaching higher values with respect to GOES-13/HEPAD increase. In addition AMS-02 extends GOES-13/HEPAD measurements over a wider range of rigidities and with higher resolution. On the other hand GOES-13/HEPAD can measure the event continuously over time, while AMS-02 is sensitive to these particles only when it is near the geomagnetic poles. In addition GOES-13/EPEAD provides measurements continuously at lower 


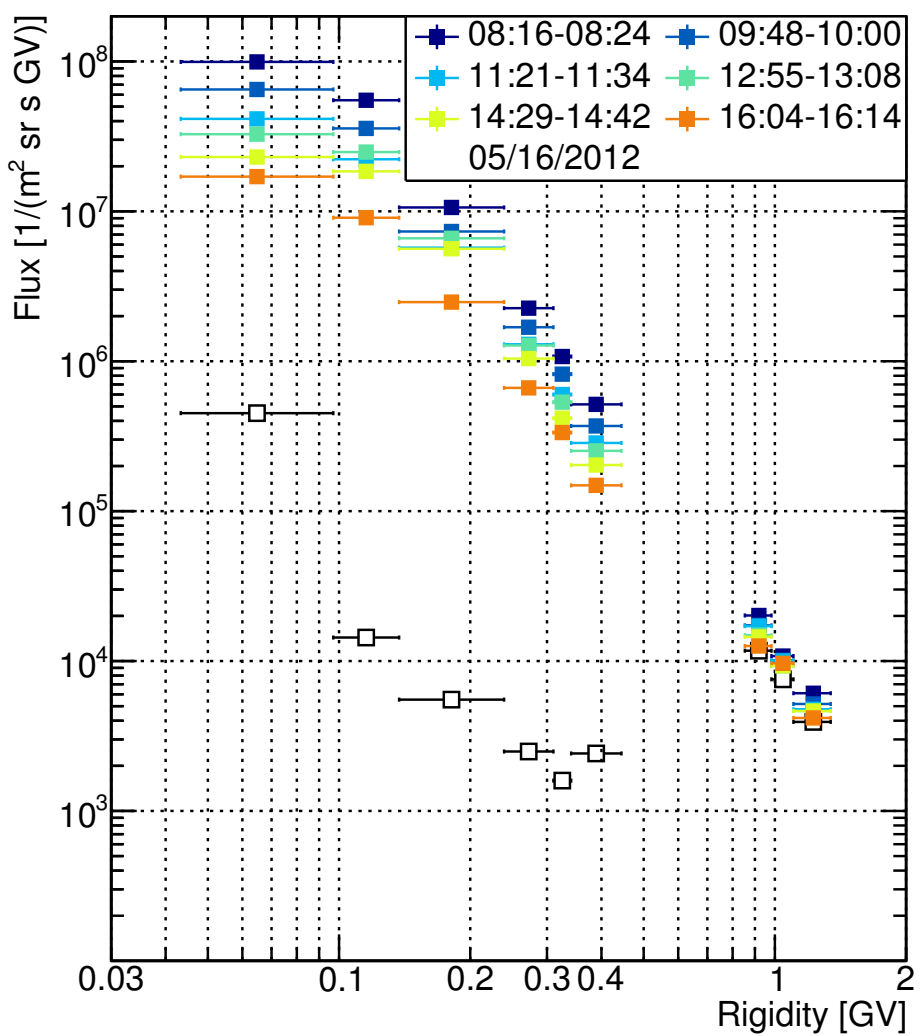

Figure 4: GOES EPEAD (below $0.5 \mathrm{GV}$ ) and HEPAD (above $0.5 \mathrm{GV}$ ) proton fluxes integrated over 24 hours measured on May 16th, 2012 (GCR background, empty squared) with various spectra measured on May 17th, 2012 integrated over short periods of time (colored squared). Protons fluxes on May 17th show a clear increase in the EPEAD channels even in a few minutes integration time.

rigidities where AMS-02 does not have access. AMS-02 and GOES-13 together provide a typical example of how multi-spacecraft observations are necessary to build a coherent picture of a solar event.

\subsection{Conclusions}

In its first three and half years of operation, AMS-02 measured an increase at low rigidities in the proton flux related to 20 major solar events associated with $\mathrm{M}$ - and X-class solar flares and fast CMEs magnetically well-connected with Earth. AMS-02, taking measurements during Solar Cycle 24 and into the next one, will generate a large sample of events that occur during different stages of the solar cycle. AMS-02 has great potential to contribute to the SEP field at high energies crucial to understand the acceleration mechanisms, with the measurement of the particle increase with extremely high statistics and with its particle identification capabilities. AMS-02 will provide high statistics and high resolution protons and helium over short periods of time, crucial to understand the time evolution of the accelerated particles. AMS-02 results used together with other experiments will represent a baseline for the theoretical models that want to describe 

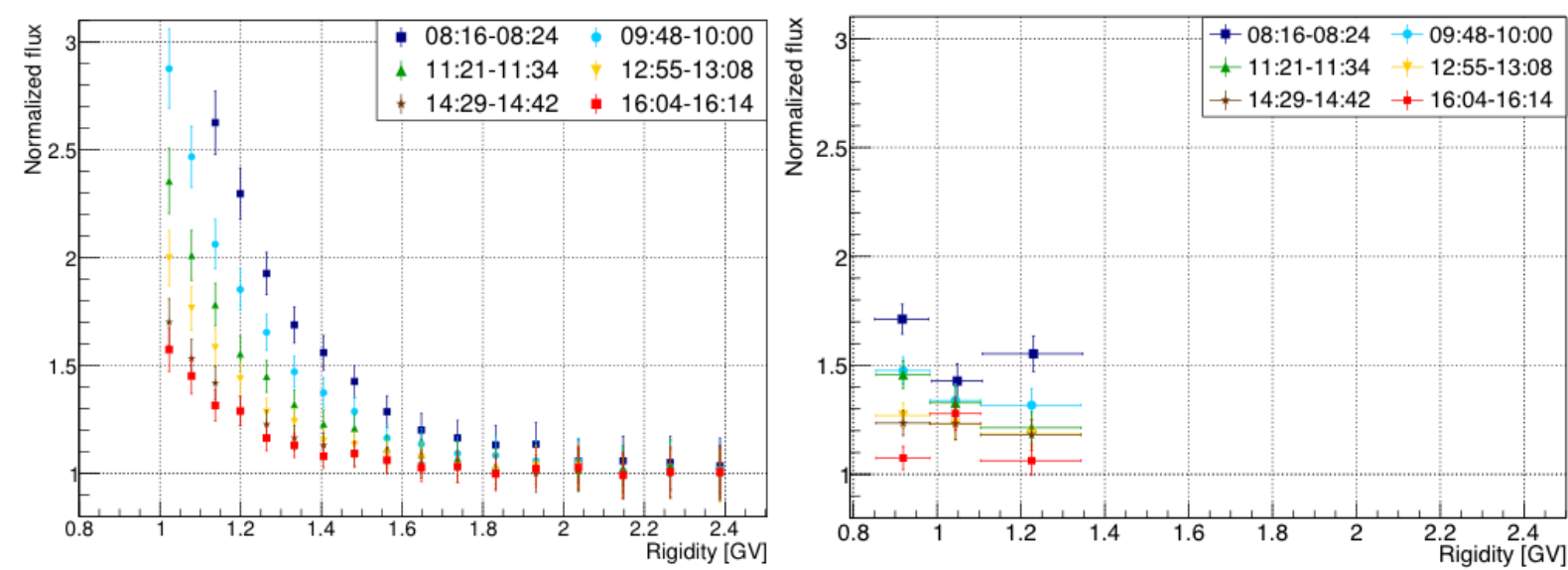

Figure 5: Left: AMS-02 protons above cutoff measured on May 17th, 2012 at different time intervals normalized to the flux measured on May 16th, 2012. Protons on May 17th show a clear increase at low rigidities even in a few minutes integration time. Right: GOES-13/HEPAD normalized proton fluxes measured in the same time intervals of AMS-02 for comparison purpose.

particles acceleration processes at the sun and their propagation inside the heliosphere and the magnetosphere.

\subsection{Acnowledgement}

This work has been supported by acknowledged person and institutions in [2] and supported by NASA Radiation Group at Jonson Space Center, Wyle Laboratories, INC. Grant: 2014/T72497. Thanks to Dr. A. Tylka for the all invaluable discussions about SEP theory and experiments and for all the input about GOES data analysis.

\section{References}

[1] M. A. Lee. Coupled hydromagnetic wave excitation and ion acceleration at an evolving coronal/ interplanetary shock. ApJS, 158:38L, 2005. doi:10.1086/428753.

[2] Precision Measurement of the Proton Flux in Primary Cosmic Rays from Rigidity 1 GV to 1.8 TV with the Alpha Magnetic Spectrometer on the International Space Station, AMS Collaboration (M. Aguilar et al.). Physical Review Letters PRL 114, 171103 (2015)

[3] International Geomagnetic Reference Field: the eleventh generation, Geophysical Journal International Vol. 183, Issue 3.

[4] AMS-02 collaboration, Leptons in near earth orbit, Phys Lett. B Vol. 484, 29 June 2000.

[5] N. Gopalswamy, et al. Major solar eruption and high energy particle events during solar cycle 24. Earth, Planets and Space 2014, 66:104

[6] Geostationary Operational Environmental Satellite. Available from: http://www.swpc.noaa.gov/Data/goes.html 